\title{
ANDES

www.scielo.cl

\section{Adiposidad corporal y resistencia muscular abdominal en jóvenes con síndrome de Down}

\author{
Body adiposity and abdominal muscle resistance in young people with Down syndrome
}

\author{
Marcelo Pino ${ }^{\mathrm{a}}$, Fernando Muñoz ${ }^{\mathrm{b}, \mathrm{h}}$, Matías Henríquez ${ }^{\mathrm{ch}, \mathrm{i}}$, Cristian Luarte Rocha ${ }^{\mathrm{d}, \mathrm{i}}$, \\ Rossana Gomez Campos ${ }^{\mathrm{e}}$, Marco Cossio Bolaños ${ }^{\mathrm{f}}$, Luís Felipe Castelli de Campos, ${ }^{\mathrm{g}, \mathrm{i}}$
}

\author{
aEscuela Nacional de Pedagogía en Educación Física, Facultad de Educación, Universidad Santo Tomás. Santiago, Chile \\ bDepartamento de Kinesiología, Universidad Metropolitana de Ciencias de la Educación. Santiago, Chile \\ Instituto Nacional de Rehabilitación Pedro Aguirre Cerda. Santiago, Chile \\ ¿Escuela de Educación Física, Facultad de Educación, Universidad San Sebastián. Concepción, Chile \\ eDepartamento de Diversidad e Inclusividad Educativa, Universidad Católica de Maule. Talca, Chile \\ 'Departamento de Ciencias de la Actividad Física, Universidad Católica del Maule. Talca, Chile \\ 9Departamento de Ciencias de la Educación, Universidad del Bío-Bío, Chillán, Chile \\ hociedad Chilena de Actividad Física Adaptada-SOCHIAFA, Chile \\ 'Grupo de Investigación en Deporte Adaptado y Paralímpico-GIDEPAUSS. Chile
}

Recibido: 22 de noviembre de 2019; Aceptado: 12 de febrero de 2021

¿Qué se sabe del tema que trata este estudio?

Diversos estudios han sugerido que la fuerza muscular y los niveles de adiposidad corporal podrían influir sobre la calidad de vida, autonomía, e independencia funcional de jovenes con y sin discapacidad, así también particularmente en jóvenes con Síndrome de Down.

\section{¿Qué aporta este estudio a lo ya conocido?}

Los jóvenes con Síndrome de Down, que exhiben niveles satisfactorios de resistencia muscular abdominal, presentaron valores disminuidos del índice de masa corporal e índice de cintura-estatura, destacándose la aplicabilidad de estas variables para la evaluación de la condición física en esta población.

\section{Resumen}

Objetivo: verificar la relación entre la resistencia muscular abdominal y la adiposidad corporal medido por el indice cintura-estatura en jóvenes con síndrome de Down (SD). Pacientes y Método: Estudio descriptivo comparativo en 115 jóvenes con SD, 65 varones, rango de edad 10 a 18 años, provenientes de la región del Maule. Se evaluó el peso, la estatura y la circunferencia de la cintura. Se calculó el índice de masa corporal (IMC) y el índice de cintura-estatura (ICE). Se evaluó la prueba de resistencia muscular abdominal (RMA) en 60 segundos y se clasificó en dos categorías (bajo RMA y aceptable RMA). Resultados: En ambos sexos los participantes clasificados con adecuados niveles de RMA presentaron menores valores de ICE e IMC $(\mathrm{p}<0,05)$, mientras que los clasificados con bajo
Palabras clave: Adiposidad Corporal; Fuerza Muscular; Síndrome de Down; Condición Física

Correspondencia:

Luis Felipe Castelli Correia de Campos

Icastelli@ubiobio.cl 
nivel de RMA evidenciaron elevados valores de ICE e IMC $(\mathrm{p}<0,05)$. Las correlaciones entre adiposidad y RMA fueron negativas y oscilaron en ambos sexos entre $r=-0,20$ a $-0,25, p<0,05$. Conclusiones El estudio demostró que los jóvenes de ambos sexos con SD clasificados con niveles adecuados de RMA, presentaron valores disminuidos de ICE.

\section{Abstract}

Objective: to verify the relationship between abdominal muscle endurance and the level of body fat, measured through the waist-to-height ratio, in young people with Down syndrome (DS). Patients and Method: A comparative descriptive study was carried out in 115 young people with DS ( $n=65$ men and $\mathrm{n}=50$ women) aged between 10 and 18 years, from the Maule Region. Age, weight, height, and waist circumference were evaluated. Body mass index (BMI) and waist-to-height ratio (WHtR) were calculated. The abdominal muscle endurance test (AME) was evaluated in 60 seconds and classified into two categories (low AME and acceptable AME). Results: Subjects of both sexes classified with adequate levels of AME showed lower WHtR and BMI values $(\mathrm{p}<0.05)$, while those classified with low levels of AME showed higher values of WHtR and BMI $(p<0.05)$. The correlations between adiposity and AME were negative and ranged in both sexes from $r=0.20$ to $0.25, p<0.05$. Conclusion: The study showed that young people of both sexes with DS classified with adequate levels of AME, presented decreased values of WHtR.
Keywords:

Body Fat;

Muscle Strength;

Down Syndrome;

Physical Fitness

\section{Introducción}

El síndrome de Down (SD) es una de las condiciones de discapacidad intelectual más prevalentes a nivel mundial ${ }^{1}$. Debido a las mejoras en tratamientos médicos y acceso a servicios de salud la expectativa de vida de los niños y niñas con este síndrome ha aumentado ${ }^{2}$. Es ampliamente conocido que las personas con SD presentan un mayor riesgo de sufrir enfermedades cardiovasculares, Alzheimer y leucemia infantil ${ }^{3}$. Además, tienen una mayor frecuencia de llevar un estilo de vida sedentario, apnea obstructiva del sueño, dislipidemia, hiperinsulinemia, dificultades en la marcha, bajos niveles de aptitud física, sobrepeso, y obesidad ${ }^{3-8}$.

La aptitud física relacionada a la salud, independientemente del tipo de población considerada (con o sin discapacidad intelectual), según Heyward ${ }^{9}$, incluye la composición corporal, capacidad aeróbica, fuerza muscular y flexibilidad. La fuerza muscular es uno de los componentes que más ha sido estudiado en niños $y$ adolescentes sin discapacidad ${ }^{10-12}$. Sin embargo, en jóvenes con discapacidad intelectual, y principalmente con SD, los estudios han sugerido que la fuerza muscular tiene gran impacto sobre la calidad de vida ${ }^{13-15}$, autonomía, e independencia funcional ${ }^{13}$.

En ese contexto, la preservación de la fuerza muscular a un nivel satisfactorio, independientemente de sus manifestaciones (por ejemplo, fuerza isométrica máxima, resistencia muscular y fuerza explosiva $)^{16}$, es necesaria para el desarrollo de las actividades diarias en jóvenes con SD. Varios estudios en niños sin discapacidad intelectual han demostrado que la fuerza muscular está asociada inversamente e independientemente con la resistencia a la insulina, riesgo metabólico, proteínas inflamatorias, adiposidad corporal durante la infancia y la adolescencia ${ }^{10,11,16,17}$. Es muy probable que el aumento de la adiposidad corporal en estas personas se relacione negativamente con la resistencia muscular abdominal, capacidad que puede ser evaluada mediante pruebas de resistencia dinámicas, (ej: realizar abdominales durante un minuto $)^{18}$.

El objetivo de este estudio fue verificar la relación entre la resistencia muscular abdominal y la adiposidad corporal, medido por el índice de cintura-estatura en jóvenes con SD. Esta información puede servir para desarrollar programas de intervención en jóvenes con bajos niveles de resistencia muscular, con riesgo de desarrollar sobrepeso y obesidad.

\section{Pacientes y Método}

Estudio transversal descriptivo y comparativo. Se estudió a 115 jóvenes con SD $(n=65$ varones y $n=50$ damas), con un rango de edad entre 10 y 18 años. Todos se encontraban integrados a tres centros escolares de educación especial de la comuna de Talca, Chile. La selección de la muestra se realizó por medio de cuotas de forma no-probabilística.

El grado de cociente intelectual de los estudiantes fue diagnosticado entre leve y/o moderado según la escala WAIS-IV para los jóvenes $\geq 17$ años y se utilizó la escala WISC-V para los participantes de edad entre los 16 años y los 11 meses $^{19,20}$. Esta información fue pro- 
porcionada por la dirección de cada escuela de donde provenían los estudiantes. Los apoderados responsables de los participantes fueron informados acerca del diseño de estudio y las variables a recolectar. De forma voluntaria todos firmaron el consentimiento informado. Este estudio se efectuó de acuerdo con la declaración de Helsinki para seres humanos y fue aprobado por el comité de ética de la Universidad Santo Tomás (Código nº ID-116).

\section{Procedimientos}

Todas las evaluaciones se realizaron en el transcurso de tres semanas consecutivas durante el mes de agosto del año 2019 durante las clases de educación física en la escuela.

Para la evaluación de las variables antropométricas de peso, estatura y circunferencia de la cintura (CC), estas fueron efectuadas con pantalón corto, polera y sin zapatos. Se utilizó el protocolo estandarizado propuesto por la Sociedad Internacional para el Avance de la Cineantropometría ${ }^{21}$. La masa corporal $(\mathrm{kg})$ se evaluó utilizando una balanza digital (Tanita, modelo SC 240-MA) con precisión de $100 \mathrm{~g}$. La estatura $(\mathrm{cm})$ se estimó mediante un estadiómetro portable (Seca, modelo 213) con precisión de $1 \mathrm{~mm}$, la CC se midió mediante una cinta métrica (Seca) con precisión de $1 \mathrm{~mm}$. Se calculó el índice de masa corporal [IMC $=$ Peso $(\mathrm{kg}) /$ estatura $\left.^{2}(\mathrm{~m})\right]$ y el índice cintura-estatura $\left.[\mathrm{ICE}=\mathrm{C} / \mathrm{E})\right]$. El estado nutricional se calculó utilizando el Z escore para el IMC $(z$-IMC) de acuerdo a la referencia propuesta por la Organización Mundial de la salud $(\mathrm{OMS})^{22}$, considerando valores de score $\mathrm{Z}<1,00$ como normopeso, 1,00 < score $Z<1,99$ como sobrepeso y score $Z>2,00$ como obeso.

Para la evaluación de la fuerza muscular se utilizó la prueba de resistencia muscular abdominal (RMA) que consistió en realizar el mayor número de repeticiones durante el tiempo de 60 segundos. Para eso, fue realizado un calentamiento previo de 10 minutos con alternancia de ejercicios de movilidad articular, flexibilidad estática y dinámica. Como protocolo, los participantes fueron instruidos a posicionarse en posición supino con rodillas flexionadas y los pies apoyados en el suelo. La prueba fue realizada sobre una colchoneta con ayuda de un compañero que sujetaba los pies. Para registrar el tiempo, se utilizó un cronómetro Casio ${ }^{\circledR}$ con una precisión de $(1 / 100 \mathrm{seg})^{23}$. Las evaluaciones se realizaron con la participación de 3 evaluadores, todos con amplia experiencia en procesos de toma de medidas y evaluaciones de la aptitud física relacionada a la salud.

Los puntos de corte para la RMA fueron considerados de acuerdo a la guía de referencia para la evaluación de la condición física ${ }^{24}$ orientada para niños y adolescentes de California (Estados Unidos). Los puntos de corte están determinados por edad y sexo para la RMA: En hombres: $\leq 12$ abdominales a los 10 años, $\leq 15$ abdominales a los 11 años, $\leq 18$ abdominales a los 12 a 18 años. En mujeres, $\leq 12$ abdominales a los 10 años, $\leq 15$ abdominales a los 11 años, $\leq 18$ abdominales a los 12 años, $\leq 21$ abdominales a los 13 años $y \leq 24$ abdominales a los 14 a 18 años.

\section{Estadística}

Para el análisis estadístico se utilizó el programa Graphpad Prism ${ }^{\circledast}$ 8. Se aplicaron evaluaciones de normalidad de los datos en base a la prueba Shapiro Wilk y la prueba de homogeneidad de varianzas en base a la prueba de Levene. Para todos los resultados se calculó la media y la desviación estándar (DE). Para las comparaciones entre las medias de ambos sexos, se utilizó test t para muestras independientes. Para la RMA se clasificó en dos grupos (bajo y aceptable) y se compararon por medio de test $t$ para muestras relacionadas. Las comparaciones entre categorías según ${ }_{Z}$-IMC fueron comparadas mediante análisis de varianza (ANOVA) de una vía y la prueba de especificidad de Tukey. Las relaciones entre variables se efectuaron a través del coeficiente de correlación de Pearson. Las prevalencias del estado nutricional se compararon con $\mathrm{X}^{2}$. El nivel de significancia considerado fue de $\mathrm{p}<0,05$.

\section{Resultados}

Las variables antropométricas, índices de adiposidad y la RMA se describen en la tabla 1 . No se encontraron diferencias significativas en la edad, circunferencia de la cintura e IMC entre ambos sexos, sin embargo, los hombres presentaron un mayor peso, estatura, $z$-IMC y RMA que las mujeres $(\mathrm{p}<0,05)$, mientras que estas últimas reflejaron un mayor ICE que sus contrapartes $(\mathrm{p}<0,05)$. No hubo diferencias significativas entre las prevalencias del estado nutricional (normopeso, sobrepeso y obesidad) entre ambos $\operatorname{sexos}\left(\chi^{2}=0,6000, p=0,7408\right)$.

Las comparaciones del ICE e IMC por categorías de la RMA se muestran en la figura 1. En ambos sexos se observaron diferencias significativas entre las dos categorías de RMA ( $p<0,05)$, tanto para el ICE e IMC. En el ICE en hombres [baja RMA 0,54 $\pm 0,06$ y aceptable RMA 0,51 $\pm 0,03$ ], mientras que, en mujeres [baja RMA 0,56 $\pm 0,04$ y aceptable RMA 0,53 $\pm 0,04$ ]. Para el IMC, en hombres [bajo RMA 25,3 $\pm 4,6 \mathrm{~kg} / \mathrm{m}^{2}$ y aceptables RMA $\left.21,3 \pm 2,8 \mathrm{~kg} / \mathrm{m}^{2}\right]$, mientras que en mujeres [baja RMA $25,4 \pm 3,5 \mathrm{~kg} / \mathrm{m}^{2}$ y aceptable RMA $\left.23,6 \pm 5,2 \mathrm{~kg} / \mathrm{m}^{2}\right]$.

Las comparaciones de la RMA por categoría nutri-

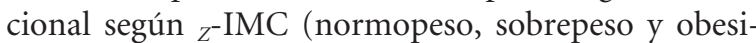
dad) se presentan en la figura 2. No hubo diferencias significativas entre las categorías de normopeso y so- 
Tabla 1. Características antropométricas y de la resistencia muscular abdominal de jóvenes con Síndrome de Down

\begin{tabular}{|c|c|c|c|c|}
\hline \multirow[t]{2}{*}{ Variables } & \multicolumn{2}{|c|}{ Hombres $(n=65)$} & \multicolumn{2}{|c|}{ Mujeres $(n=50)$} \\
\hline & $\bar{x}$ & $\mathrm{DE}$ & $\bar{x}$ & $\mathrm{DE}$ \\
\hline Edad (años) & 14,0 & 2,6 & 14,5 & 2,5 \\
\hline Peso $(k g)$ & $53,8^{*}$ & 12,7 & 49,9 & 12,8 \\
\hline Estatura $(\mathrm{cm})$ & $148,4^{*}$ & 9,3 & 143,5 & 9,8 \\
\hline IMC $\left(\mathrm{kg} / \mathrm{m}^{2}\right)$ & 24,2 & 4,6 & 24,0 & 4,9 \\
\hline$z$ IMC & $1,29 *$ & 1,0 & 1,07 & 1,26 \\
\hline $\mathrm{CC}(\mathrm{cm})$ & 78,6 & 9,3 & 79,4 & 9,1 \\
\hline ICE (u.a) & $0,53^{*}$ & 0,06 & 0,56 & 0,08 \\
\hline RMA (rep) & 14,9 * & 7,0 & 12,6 & 6,5 \\
\hline Baja RMA & 11,9 & 4,3 & 10,1 & 3,7 \\
\hline Aceptable RMA & 23,3 & 6,4 & 22,9 & 4,8 \\
\hline Estado nutricional (z-IMC) & $\mathrm{N}$ & $\%$ & $n$ & $\%$ \\
\hline Normopeso & 26 & 40 & 20 & 40 \\
\hline Sobrepeso & 27 & 42 & 19 & 38 \\
\hline Obesidad & 12 & 18 & 11 & 22 \\
\hline
\end{tabular}

x.: Promedio; DE: Desviación estándar; Min: Mínimo; Max: Máximo; IMC: Índice de masa corporal; CC: Circunferencia Cintura; ICE: Índice cintura estatura; RMA: Resistencia muscular abdominal; Rep: Repeticiones; ${ }^{*}$ Diferencia significativa en relación a las mujeres; $\chi^{2}=0,6000$; $p=0,7408$. brepeso en ambos sexos $(\mathrm{p}>0,05)$, sin embargo, estas dos categorías (normopeso y sobrepeso), tanto en hombres, como en mujeres, difieren con los clasificados con obesidad $(\mathrm{p}<0,05)$. En hombres, los valores medios de RMA en la categoría de normopeso es de $15,7 \pm 7,5$ abdominales, en sobrepeso es de $16 \pm 6,4$ abdominales y en obesidad fue de 10,6 $\pm 4,2$ abdominales, mientras que, en mujeres, en la categoría normopeso fue de 14,2 $\pm 5,1$ abdominales, en sobrepeso $14,5 \pm 6,5$ abdominales y en obesidad fue de 9,0 $\pm 5,1$ abdominales.

Las relaciones entre el ICE con la prueba de RMA en ambos sexos se presentan en la figura 3. En hombres y mujeres las correlaciones fueron negativas y bajas, pero significativas (hombres: $r=-0,20, p<0,05$, mujeres: $\mathrm{r}=-0,25, \mathrm{p}<0,05)$.

\section{Discusión}

Los resultados de este estudio han demostrado que hubo diferencias significativas en la adiposidad corporal expresada por medio del ICE cuando fueron clasificados según RMA, además, hubo relación negativa entre el ICE con la RMA en ambos sexos.

Los jóvenes de ambos sexos clasificados con mejor RMA presentaron valores inferiores de ICE en relación a sus similares con baja RMA, además, los jóvenes clasificados con obesidad según z-IMC reflejaron menor desempeño en la RMA. Estos hallazgos confirman que la aptitud física y en especial la fuerza de resistencia

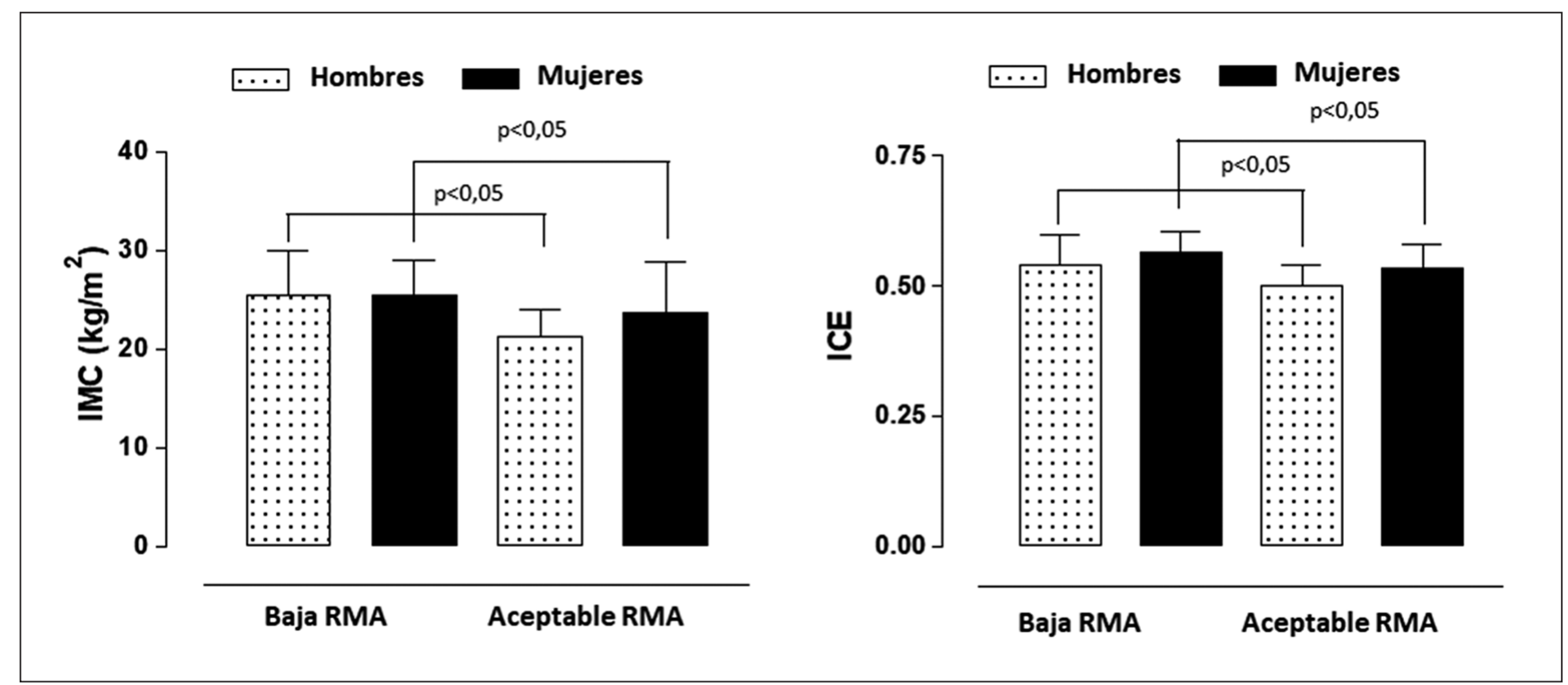

Figura 1. Comparación de los valores medios del índice de masa corporal (IMC) e Índice cintura estatura (ICE) en función de las categorías de la resistencia muscular abdominal (RMA) en jóvenes con síndrome de Down. IMC: Índice de masa corporal, ICE: Índice cintura estatura, RMA: Resistencia muscular abdominal, $p=$ diferencia significativa en relación a baja RMA. 


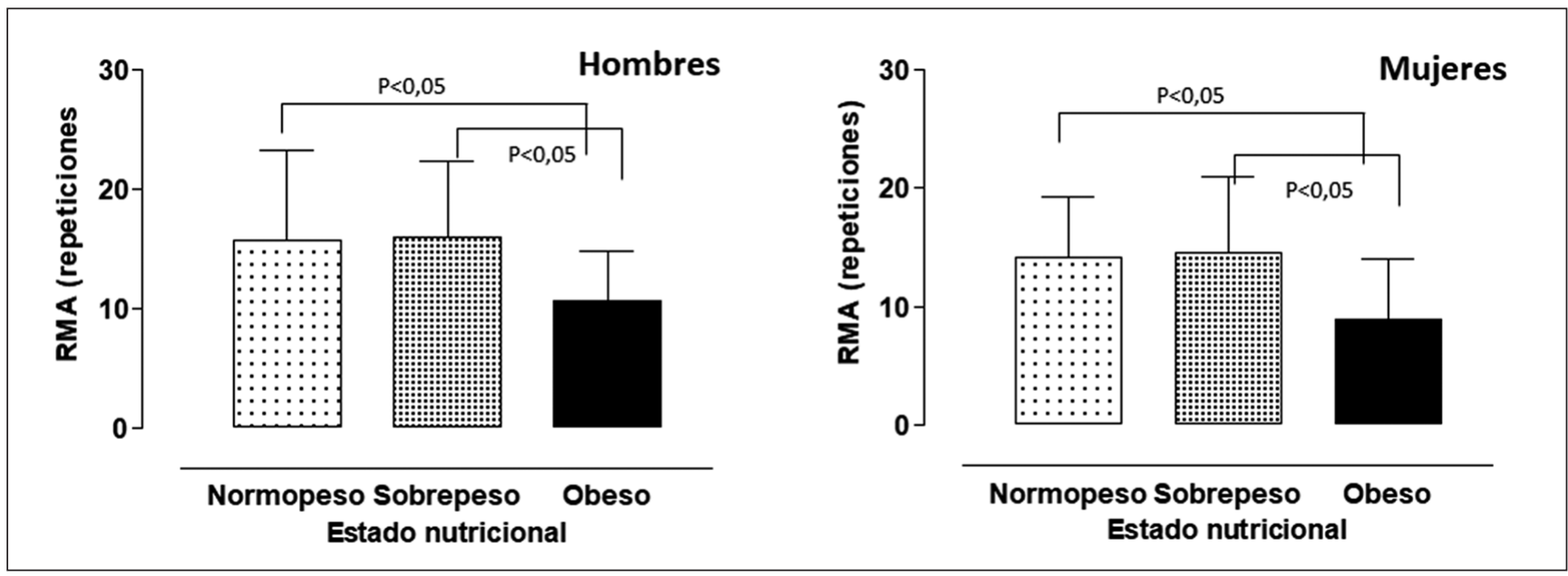

Figura 2. Comparación de los valores medios de la RMA según categorías nutricionales (z-IMC) en jóvenes con síndrome de Down. RMA: Resistencia muscular abdominal, $\mathrm{p}=$ diferencia significativa en relación a baja RMA.

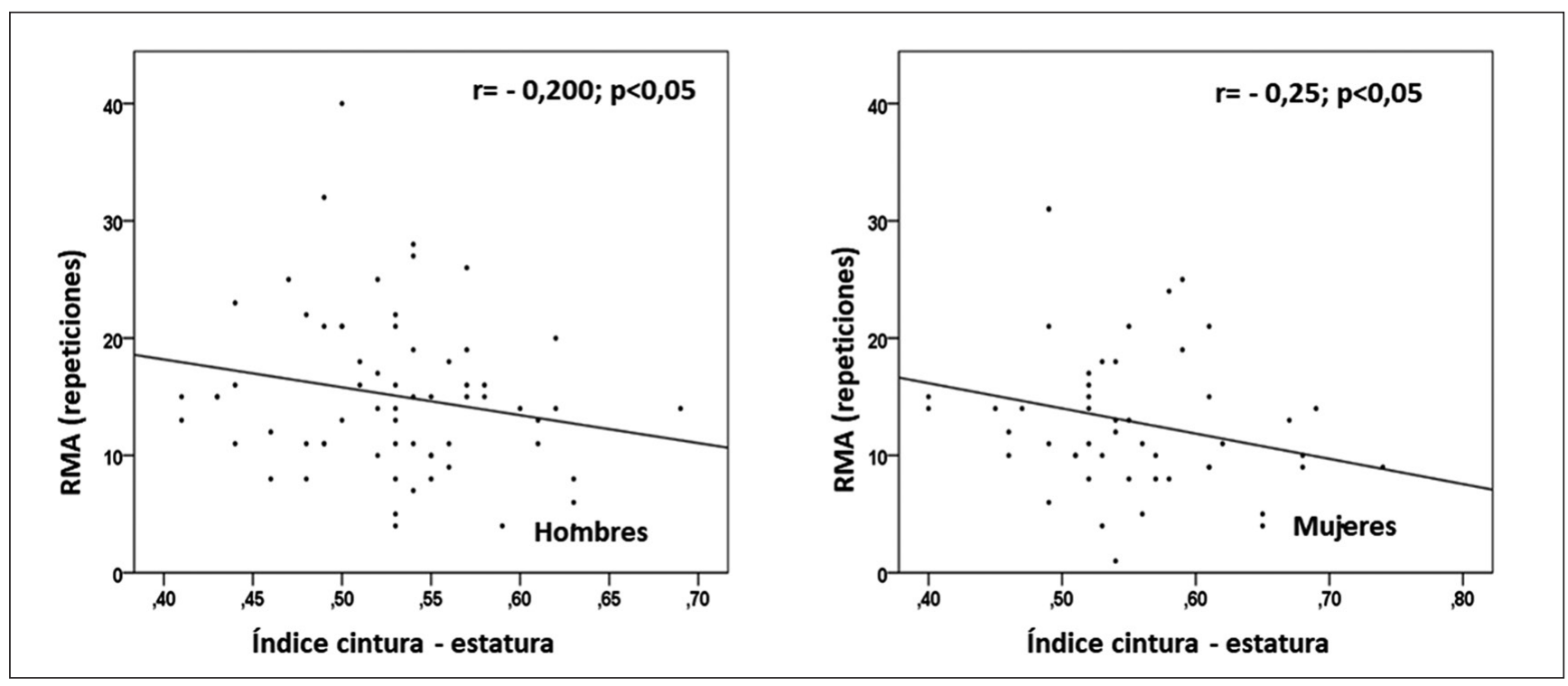

Figura 3. Relación entre la índice cintura estatura (ÍCE) con la resistencia muscular abdominal (RMA) en jóvenes con síndrome de Down.

muscular es relevante y está asociada a una reducción del acúmulo de la adiposidad corporal en la región abdominal de jóvenes con SD.

De hecho, estos hallazgos son consistentes con otros estudios efectuados en niños y adolescentes con discapacidad intelectual, donde han reportado que bajos niveles de aptitud física se relacionan negativamente con la salud general ${ }^{25-28}$. Otros estudios también han indicado que la disminución de la fuerza muscular tiene implicancias relevantes en la vida diaria y son esenciales para realizar actividades cotidianas ${ }^{29}$, por lo que al parecer la combinación de bajos niveles de actividad física y un alto nivel de sedentarismo podría tener una mayor implicancia ${ }^{30}$ en el desempeño de la fuerza muscular, respectivamente.

Es importante evaluar la aptitud muscular en jóvenes con SD, en especial la RMA, puesto que bajos niveles de fuerza y resistencia muscular podrían limitar la independencia funcional en la edad adulta ${ }^{31}$. Además, la evaluación y monitorización de los niveles de fuerza durante la etapa de crecimiento debe ser un factor prioritario. Una detección temprana de bajos niveles de fuerza muscular en poblaciones con SD puede ayudar a detectar insuficiencias en el mantenimiento de la estabilidad y consecuentemente en el equilibrio postural $^{32}$. 
Tras los resultados obtenidos en este estudio, se destaca que es necesario un mayor análisis y comprensión de las dimensiones de la fuerza muscular en poblaciones con SD, puesto que futuros estudios podrían ayudar a identificar y seleccionar las pruebas de fuerza muscular que mejor se ajusten y se adapten en niños, jóvenes y adultos, aunque evaluar la aptitud muscular de forma precisa en niños con obesidad puede ser particularmente desafiante ${ }^{29}$. En ese sentido, estos correlatos corroboran con otros estudios que se debe apuntar a desarrollar programas de intervención que promuevan la salud, incrementando la actividad física en adolescentes con SD puesto que esto puede contribuir al mejoramiento de los niveles de aptitud física general en jóvenes con obesidad en la educación escolar ${ }^{28,33}$, con especial atención en el desarrollo de ejercicios de fuerza muscular, ya que están asociados a la mejora la aptitud muscular para la realización de movimientos repetitivos y cotidianos ${ }^{34}$. Estos ejercicios podrían jugar un papel relevante para mejorar los niveles de aptitud muscular de los jóvenes con SD. Estas actividades podrían aplicarse por medio del diseño de guías o recomendaciones desde edades tempanas, puesto que podría tener influencia en el largo plazo y ser implementadas en la familia, en terapias estructuradas, en programas de intervención y en las clases de educación física ${ }^{35}$.

El estudio presenta algunas debilidades que deben ser reconocidas, dado que durante la investigación se diseñó un estudio transversal, en el que no se pueden establecer relaciones causales, además no se pudo evaluar los niveles de actividad física y el tipo de alimentación, por lo que los resultados deben ser analizados con precaución. Por el contrario, sin perjuicio de lo anterior, el estudio sirve como línea de base para desarrollar futuras comparaciones y al mismo tiempo es una de las primeras investigaciones efectuadas en la región del Maule, el cual brinda información relevante que puede ser utilizada para el desarrollo de políticas públicas locales.

Como aplicación práctica del estudio, identificar las diferencias de adiposidad corporal y el nivel de fuerza entre individuos con SD de diferentes edades y géneros contribuye a una intervención más eficiente y menos subjetiva tanto en los aspectos nutricionales como en la prescripción de actividad física, ya que presentan particularidades específicas que requieren una intervención más individualizada.

\section{Conclusión}

Este estudio demostró que los jóvenes de ambos sexos con SD clasificados con moderados y elevados niveles de resistencia muscular abdominal reflejaron valores menores de adiposidad corporal (ICE). Estos hallazgos sugieren que en los programas de actividad física desarrollados para los jóvenes con SD consideren la realización de ejercicios físicos para la resistencia y la fuerza muscular, ya que el estudio presentó evidencias de que hay menores niveles de adiposidad corporal en jóvenes con SD con adecuados niveles de RMA en ambos sexos, así como la necesidad de desarrollar más investigaciones que puedan confirmar los resultados aquí presentados.

\section{Responsabilidades Éticas}

Protección de personas y animales: Los autores declaran que los procedimientos seguidos se conformaron a las normas éticas del comité de experimentación humana responsable y de acuerdo con la Asociación Médica Mundial y la Declaración de Helsinki.

Confidencialidad de los datos: Los autores declaran que han seguido los protocolos de su centro de trabajo sobre la publicación de datos de pacientes.

Derecho a la privacidad y consentimiento informado: Los autores han obtenido el consentimiento informado de los pacientes y/o sujetos referidos en el artículo. Este documento obra en poder del autor de correspondencia.

\section{Conflicto de intereses}

Los autores declaran no tener conflicto de intereses.

\section{Referencias}

1. Maulik PK, Mascarenhas MN, Mathers $\mathrm{CD}$, et al. Prevalence of intellectual disability: A meta-analysis of populationbased studies. Res Dev Disabil. 2011;32(2):419-36. doi: 10.1016/j. ridd.2010.12.018.

2. Pitetti K, Baynard T, Agiovlasitis S. Children and adolescents with Down syndrome, physical fitness and physical activity. J Sport Health Sci. 2013;2(1):4757. doi: https://doi.org/10.1016/j. jshs.2012.10.004.

3. Nixon DW. Down syndrome, obesity, alzheimer's disease, and cancer: A brief review and hypothesis. Brain Sci. 2018;8(4):1-14. doi: 10.3390/ brainsci8040053.

4. Bertapelli F, Pitetti K, Agiovlasitis S, et al. Overweight and obesity in children and adolescents with Down syndrome-prevalence, determinants, consequences, and interventions: A literature review. Res Dev Disabil. 2016;57:181-92. doi: 10.1016/j. ridd.2016.06.018.

5. Guerra M, Llorens N, Fernhall B. Chronotropic incompetence in persons with down syndrome. Arch Phys Med 
Rehabil. 2003;84:1604-08. doi: 10.1053/ s0003-9993(03)00342-3.

6. Alvear F, Gomez-Campos R, UrraAlbornoz C, et al. Predictores de los Indicadores de adiposidad corporal por edad cronológica y biológica en niños y adolescentes que residen en el sur de Chile. Rev Esp Nutr Hum Diet. 2017;21(4):360-8. doi: http://dx.doi. org/10.14306/renhyd.21.4.401

7. Pastore E, Marino B, Calzolari A, et al. Clinical and cardiorespiratory assessment in children with Down syndrome without congenital heart disease. Arch Pediatr Adolesc Med. 2000;154(4):408-10. doi: 10.1001/archpedi.154.4.408.

8. Wee SOT, Pitetti KH, Baynard T, et al. Impact of obesity and Down syndrome on maximal heart rate and work capacity in youth and adults with intellectual disabilities. Med Sci Sports Exerc. 2013;45(5):32-32. doi: http://hdl.handle. net/10057/10736

9. Heyward VH. Advanced Fitness Assessment \& Exercise Prescription, 5th ed. Champaign, Illinois: Human Kinetics, 2006, p. 278.

10. Ortega FB, Ruiz JR, Castillo MJ, et al. Physical fitness in childhood and adolescence: a powerful marker of health. Int J Obes. 2008;32:1-11. doi: https://doi. org/10.1038/sj.ijo.0803774.

11. Cohen DD, Gomez-Arbelaez D, Camacho $\mathrm{PA}$, et al. Low muscle strength is associated with metabolic risk factors in Colombian children: the ACFIES study. PLoSOne. 2014;9(4):e93150. doi: 10.1371/ journal.pone.0093150.

12. Plowman, SA. Muscular strength, endurance, and flexibility assessments. In S. A. Plowman \& M. D. Meredith (Eds.), Fitnessgram/Activitygram reference guide (4th ed., pp. 8-1-8-55). Dallas, TX: Cooper Institute. 2010.

13. Gonzalez-Agüero A, Vicente-Rodriguez G, Moreno A, et al. Health-related physical fitness in children and adolescents with Down syndrome and response to training. Scand J Med Sci Sports. 2010;20:716-24. doi: 10.1111/j.1600-0838.2010.01120.x.

14. Croce RV, Pitetti KH, Horvat M. Peak torque, average power, and hamstrings/ quadriceps ratios in nondisabled adults and adults with mental retardation. Arch Phys Med Rehabil. 1996;77:369-72. doi: 10.1016/s0003-9993(96)90086-6.
15. Legerlotz K. The effects of resistance training on health of children and adolescents with disabilities. Am J Lifestyle Med. 2018;14(4):382-96. doi: https://doi. org/10.1177/1559827618759640.

16. Fernández-Santos J, GonzalezMontesinos JL, Ruiz J, et al. Kinematic analysis of the standing long jump in children 6- to 12-years-old. Meas Phys Educ Exerc Sci. 2018;22(1): 70-8. Doi: 10.1080/1091367X.2017.1383913.

17. Sepúlveda X, Méndez Cornejo J, Duarte Farfán C, et al. Relationship between body adiposity and horizontal jump in schoolchildren and adolescents. Rev Chil Pediatr. 2018; 89(6):701-8. doi: 10.4067/ S0370-41062018005001003.

18. Van Praagh E. Testing anaerobic performance. In: Bar OR, editor. The child and adolescent athlete. Oxford: Blackwell Scientific Publications, IOC Medical Commission; 1996.64) Faigenbaum AD, Milliken LA, Westcott WL. Maximal strength testing in healthy children. J Strength Cond Res. 2003;17:162-6.

19. Wechsler D. WAIS-IV. Escala de inteligencia de Wechsler para adultosIV. Manual técnico y de interpretación. Madrid: NCS Pearson, 2012

20. Wechsler D. WISC-V. Escala de inteligencia de Wechsler para niños-V. Madrid: Pearson, 2015.

21. International Society for the Advancement of Kinanthropometry (ISAK). International Standards for Anthropometric Assessment. Nueva Zelanda. 2001

22. Growth reference data for 5-19 years. WHO reference 2007. Extraído January 8th 2016. Disponible en: http://www.who. int/growthref/en/.

23. Soares V, Sessa M. Medidas de força muscular. En: Matsudo VKR. Testes de ciência do esporte. 2da Ed. São Caetano do Sul: CELAFISCS. 1983:57-68.

24. California Physical Fitness Test (PFT): Reference Guide. California Department of Education, USA, 2020. https://pftdata. org/files/Reference_Guide.pdf

25. Ruiz JR, Castro-Piñero J, Artego EG, Ortega FB, Sjöström M, Suni J et al. Predictive validity of health-related fitness in youth: a systematic review. Br J Sports Med. 2009;43:909-23. doi: 10.1136/ bjsm.2008.056499

26. Izquierdo-Gomez R, Martinez-Gomez
D, Tejero-Gonzalez CM, et al. Are poor physical fitness and obesity two features of the adolescent with Down syndrome? Nutr Hosp. 2013;28:1348-51. doi: 10.3305/nh.2013.28.4.6566.

27. Hartman E, Smith J, Westendorp M, et al. Development of physical fitness in children with intellectual disabilities. J Intellect Disabil Res. 2015;59(5):439-49. doi: $10.1111 /$ jir.12142.

28. Wouters M, Evenhuis HM, Hilgenkamp T. Physical fitness of children and adolescents with moderate to severe intellectual disabilities. Disabil Rehabil. 2019;42(18):1-11. doi: 10.1080/09638288.2019.1573932.

29. Thivel D, Ring-Dimitriou S, Weghuber $\mathrm{D}$, et al. Muscle strength and fitness in pediatric obesity: a systematic review from the European childhood obesity group. Obes Facts. 2016;9:52-63. doi: $10.1159 / 000443687$.

30. Medonca CV, Pereira FD, Fernhall BO. Reduced exercise capacity in persons with Down syndrome: cause, effect and management. Ther Clin Risk Manag. 2010;8:601-10. doi: 10.2147/TCRM. S10235

31. Oppewal A, Hilgenkamp TIM, van Wijck $\mathrm{R}$, et al. Physical fitness is predictive for a decline in daily functioning in older adults with intellectual disabilities: results of the HAID study. Res Dev Disabil. 2014;35:2299-315. doi: 10.1016/j. ridd.2014.05.027.

32. Shumway-Cook A, Woollacott MH. Dynamics of postural control in the child with Down syndrome. Phys Ther. 2011;65:1315-22. doi: 10.1093/ ptj/65.9.1315.

33. Garcia-Vicencio S, Coudeyre E, Kluka V, et al. The bigger, ¿the stronger? Insights from muscle architecture and nervous characteristics in obese adolescent girls. Int J Obes. 2015;40:1-7. doi: https://doi. org/10.1038/ijo.2015.158

34. Elsawy, G. Effect of Functional Strength Training on Certain Physical Variables and Kick of Twimeo Chagi among Young Taekwondo Players. World J Sport Sci. 2010;3:683-6.

35. Barr, M, Shields, N. Identifying the barriers and facilitators to participation in physical activity for children with down syndrome. J Intellect Disabil Res. 2011;55(2):1020-33. doi: 10.1111/j.13652788.2011.01425.x. 